\title{
Identification of Trace 2-Chloropropene with a New Chirped Pulse Microwave Spectrometer
}

\author{
Z. KISIEL* AND J. KOSARZEWSKI
}

Institute of Physics, Polish Academy of Sciences, Aleja Lotnikow 32/46, PL-02668 Warsaw, Poland

(Received February 13, 2017)

The design of a new chirped pulse, Fourier-transform microwave spectrometer for recording broadband rotational spectra of supersonic expansion samples is described. The spectrometer can record spectral windows of width of up to $2.2 \mathrm{GHz}$ over the $2-18 \mathrm{GHz}$ frequency region. It is complemented by a new FFT program with several novel features. Tests of the spectrometer revealed the presence of an initially unknown volatile trace molecule in a sample of 2,2-dichloropropane (DCP). The broadband nature of the new spectrometer allowed rapid assignment of the new species as 2-chloropropene, $\mathrm{CH}_{3} \mathrm{CCl}=\mathrm{CH}_{2}(2 \mathrm{CP})$. Additional $\mathrm{mm}$ wave measurements combined with analysis of previous DCP spectra up to $311 \mathrm{GHz}$ allow us to report the hitherto most precise spectroscopic constants for $2 \mathrm{CP}$.

DOI: 10.12693/APhysPolA.131.311

PACS/topics: chirped pulse, Fourier transform microwave, supersonic expansion, millimetre wave, rotational spectroscopy

\section{Introduction}

The development by Balle and Flygare [1] of the cavity, the Fourier-transform microwave (FTMW) rotational spectroscopy of supersonic expansion opened new horizons in precise studies of isolated molecules and of intermolecular complexes [2]. A modified version of a spectrometer of that type constructed in this laboratory [3] has been used for many such studies, including those of the hydrogen bonded clusters $\left(\mathrm{H}_{2} \mathrm{O}\right)_{2} \mathrm{HCl}[4]$ and $\left(\mathrm{H}_{2} \mathrm{O}\right)_{2} \mathrm{HBr}$ [5], the molecules pyrimidine [6], anisole and benzaldehyde [7], and the natural product camphor [8].

The advantages of the cavity FTMW method, such as high resolution and sensitivity, are in many applications offset by the very narrow spectral window (typically less than $1 \mathrm{MHz}$ ) available during a single measurement. This necessitates time consuming scanning procedures in cases of unknown rotational spectra. This disadvantage has been removed by the more recent experimental technique developed by the Pate research group [9].

The key new feature is to dispense with the frequency limiting resonator and to use simple microwave illumination of the studied sample by means of direct propagation between two broadband microwave antennas. The power requirements necessary to obtain broadband frequency coverage by means of a simple rectangular exciting pulse become prohibitive, but excitation was made possible by using a longer, chirped microwave pulse containing an internal frequency sweep. Direct detection of the molecular free induction decay (FID) signal emitted by the excited sample was then carried out with a fast digital oscilloscope. The signals are very weak so that it is advantageous to maximise the power of the exciting pulse, requiring the use of a multi watt power amplifier $(300 \mathrm{~W}$ in [9]). The response signal is still very weak so that

*corresponding author; e-mail: kisiel@ifpan.edu.pl averaging over many hours or days is necessary. The original chirped pulse spectrometer design has been refined further $[10,11]$ in order to overcome various instrumental challenges and this enabled many spectacular studies, such as those of the small water clusters [11-14].

Several modifications of the Pate lab chirped pulse spectrometer design have already been reported, with the aim of achieving lower construction costs by reducing the instantaneous bandwidth and limiting the operational region to $6-18 \mathrm{GHz}$ or $8-18 \mathrm{GHz}$. Some examples are the spectrometers developed at Amherst [15] and Manitoba [16] (both $6 \mathrm{GHz}$ bandwidth), North Texas [17, 18] (2 GHz bandwidth), and Eastern Illinois [19] (two overlapped $230 \mathrm{MHz}$ sidebands).

Presently, we report the construction of a chirped pulse spectrometer that allows flexible detection over the whole 2-18 GHz frequency range and is integrated with the existing cavity FTMW spectrometer [3]. The testing phase of this spectrometer already delivered an unexpected molecular result and this is also described further below.

\section{Experimental details}

\subsection{The chirped pulse spectrometer}

The new spectrometer was constructed according to two principal design criteria: flexibility of operation and integration with the existing cavity FTMW spectrometer. The latter enabled a significant reduction in the construction costs by using the same high vacuum chamber and pumping for both configurations. Furthermore, flexible design not only ensured relatively easy switch between the cavity and chirped pulse modes, but also provided chirped pulse access to the whole $2-18 \mathrm{GHz}$ frequency region (normally covered by means of two separate spectrometers). This was complemented by programmable frequency width of the chirped pulse, which allowed various trade-offs between width of coverage and sensitivity. The newly constructed chirped pulse spectrometer is shown in a simplified schematic diagram in Fig. 1. 


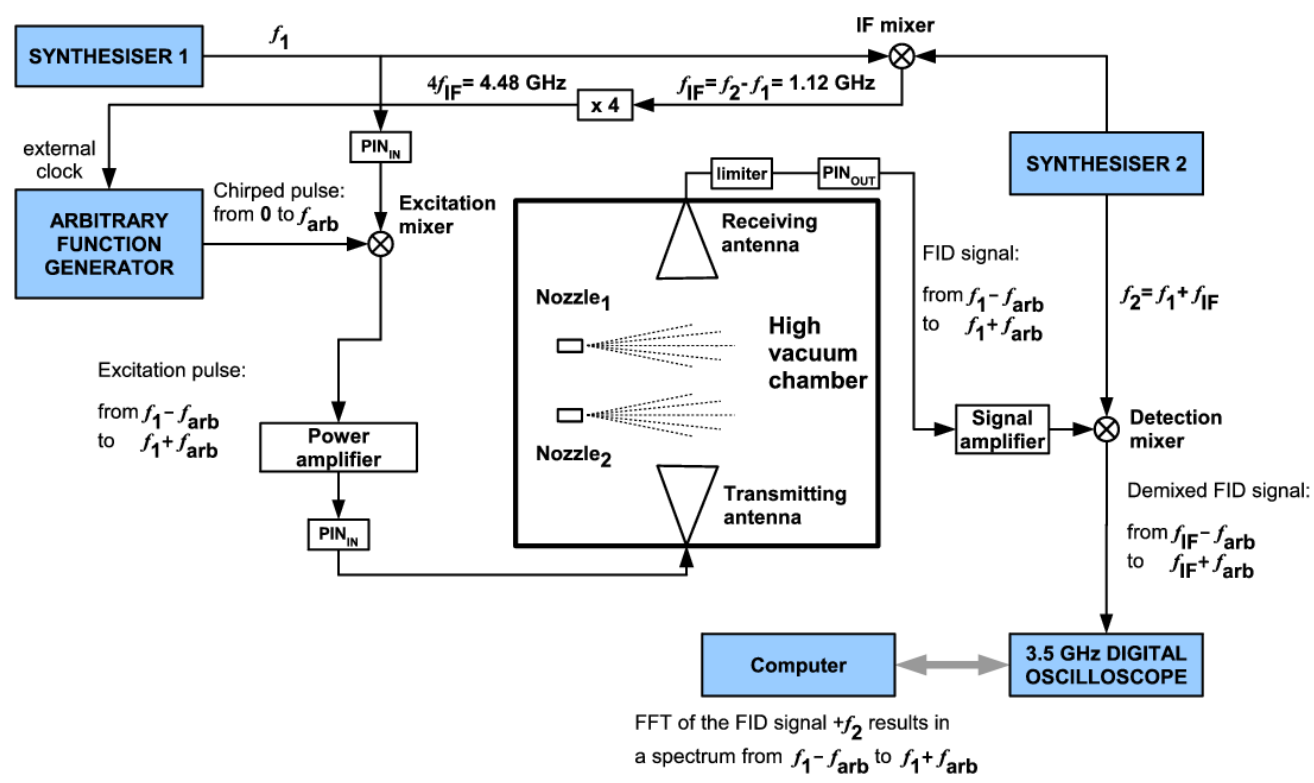

Fig. 1. The newly constructed chirped pulse spectrometer at IFPAN. The spectrometer is designed to record a spectral window of width of up to $2.2 \mathrm{GHz}$, which is movable over the $2-18 \mathrm{GHz}$ frequency region. Only two elements, the input power amplifier and the signal amplifier, need to be changed in order to cover the the whole operational frequency region. The operational frequencies in various parts of the spectrometer are indicated.

The chirped pulse measurement option has been incorporated in the existing cavity spectrometer along an axis of the chamber that is perpendicular to that of the Fabry-Perot resonator. In the cavity configuration this axis was reserved for the Stark measurements [20] and can still be reused for that purpose when necessary. Two broadband horn antennas (Q-par Angus WBH218S) covering $2-18 \mathrm{GHz}$ are used and supersonic expansion is from up to three Parker Hannifin Series 9 pulsed valves, mounted in parallel. The microwave electronics involves a double synthesiser design (Agilent N5183A and HP $83711 B$ ). The chirp signal is programmed into and generated by a $4.6 \mathrm{GS} / \mathrm{s}$ arbitrary waveform generator (Agilent $81180 \mathrm{~B}$ ). This signal covers the frequency range from 0 to $f_{\text {arb }} \leq 1.12 \mathrm{GHz}$ and is then up-converted to the central frequency, $f_{1}$, of the first microwave synthesiser. As a result the sample is illuminated by two sidebands, covering the frequency range $f_{1}-f_{\text {arb }}$ to $f_{1}+f_{\text {arb }}$. Phase stability of the microwave detection is crucial to efficient signal averaging over long periods of time. This is ensured by using the intermediate frequency signal of $1.12 \mathrm{GHz}$ between the two synthesisers as a trigger for the oscilloscope and (when multiplied by 4 ) as a $4.48 \mathrm{GHz}$ external clock signal for the arbitrary function generator. Several different broadband power amplifiers with outputs ranging from $3 \mathrm{~W}$ to $50 \mathrm{~W}$ can be used for amplifying the microwave excitation signal. Various protective and signal forming PIN diode microwave switches are used in typical positions for a spectrometer of this type. An additional high power PIN switch just before the transmitting antenna was found to considerably improve signal to noise performance by preventing reflections back from the chamber.
The operational parameters for the supersonic expansion were adopted from the cavity mode [4] and allowed us to typically record a molecular response signal of $1 \mathrm{~ms}$ duration for a single supersonic expansion pulse. This consists of back-to-back FID responses to (usually) 20 consecutive exciting microwave pulses. The whole recorded data segment is $5 \mathrm{M}$ points long (recorded at $5 \mathrm{Gs} / \mathrm{s}$ ) and is averaged on a fast digital oscilloscope (LeCroy WavePro735Zi-A) at rate of up to $5 \mathrm{~Hz}$. The averaging is carried out for as long as necessary and the averaged waveform can be saved at any moment. Further coaddition to a single FID (50 $\mu$ s long for 20 exciting microwave pulses) is carried out within the FFTS program (see below). The number of microwave pulses and their spacing can be varied over a broad range, allowing the choice between a larger number of exciting microwave pulses and a relatively short FID for optimum sensitivity, or fewer exciting pulses and a long FID for increased resolution. A single 8-channel programmable pulse generator (Quantum Composers 9528) is sufficient to generate the pulse trains required for operation of the spectrometer in both the chirped pulse and the cavity modes.

Spectrometer operation uses a distributed computing approach. One computer is dedicated to running the program for setting up and controlling spectrometer operation by means of the GP-IB interface. The composite response signal as directly averaged in the oscilloscope is first saved on its internal hard disk, and is then transmitted over an Ethernet connection to a second computer for further processing. The consecutive FID responses in the (typically $1 \mathrm{~ms}$ long, $5 \mathrm{M}$ point) record can then be coadded, and the final molecular FID transformed to the frequency domain using various options available in 
the FFTS program. This approach prevents any interruptions to spectrometer operation from computer intensive tasks associated with managing and transforming multi-megapoint data files.

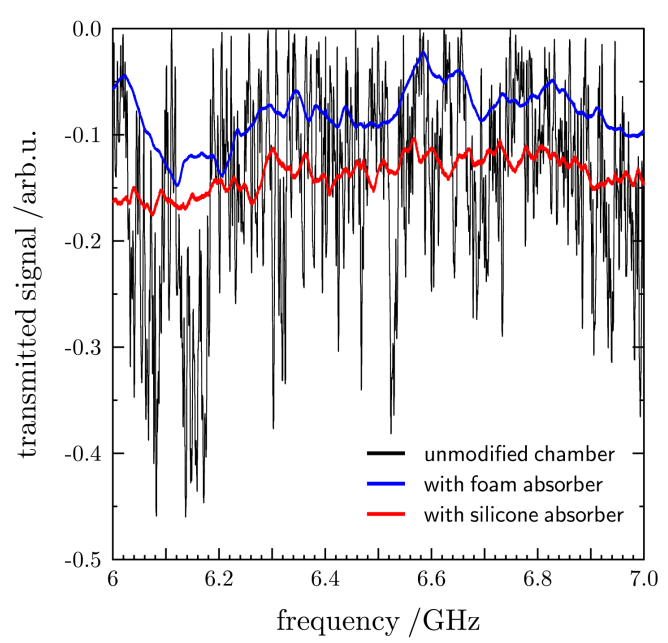

Fig. 2. Illustration of the very sharp background resonances affecting horn to horn transmission in a $60 \mathrm{~cm}$ diameter chamber containing the Fabry-Perot resonator with $50 \mathrm{~cm}$ diameter mirrors used for cavity measurements.

\subsection{Background resonances}

The decision to reuse the vacuum chamber of the cavity spectrometer produced one unexpected result, in that it turned out that the horn to horn transmission on chirped pulse operation was beset by a multitude of very sharp resonances, as illustrated in Fig. 2. While the resonance spikes were not prohibitive to broadband excitation of the sample, their high dynamic range was sufficient to significantly perturb relative intensities of measured lines and was also conducive to the appearance of spurious peaks on the frequency domain spectra resulting from the FFT. This phenomenon was known to the chirped pulse community, but it was only mentioned in passing in the literature and various ad hoc solutions were used for its attenuation.

In the present case we first used two large $60 \times 60 \mathrm{~cm}^{2}$ sheets of the foam microwave absorbent material Eccosorb RF-RET to simply cover the two mirror surfaces of the microwave resonator. This produced a satisfactory result, shown in Fig. 2, except for extremely long pumping down times after atmospheric exposure mainly due to water desorption from the very large surface area of the foam. A much improved version of resonance attenuation was finally achieved with Eccosorb FGM-125 microwave absorber material in the form of silicone sheets loaded with a suitable microwave absorbing filler. The silicone absorber shows very small desorption but is somewhat fragile so it was attached to suitable aluminum supporting plates, that were designed in a way allowing insertion and removal through the $15 \mathrm{~cm}$ access port to the chamber. Eventually, it turned out to be advantageous to shield not only the two $50 \mathrm{~cm}$ diameter Fabry-Perot resonator mirrors (oriented parallel to the axis joining the two horn antennas), but also the support rods connecting the mirrors, and the parts of the chamber nearest the horn antennas. The final result is documented in Fig. 2 and it ensured satisfactory operation over the complete 2$18 \mathrm{GHz}$ frequency range. We have recently published an extensive analysis of the rotational spectrum of the DCP molecule [21] so it was natural to use this molecule for calibration of the performance of the new spectrometer. A spectrum obtained near the low frequency limit of the new spectrometer is shown in Fig. 3. The spectrum documents very useful performance concerning the baseline, resolution and sensitivity. The spectrum was recorded in $40 \mathrm{~min}$, while investigation of the same frequency region to the same level of detail by using the cavity mode would take several days.

\subsection{The computer program FFTS}

The new spectrometer was developed in parallel with the computer program FFTS, which embodies many features that are not only of direct relevance to the current spectrometer but are also of general use for analysis of broadband Fourier-transform microwave spectra. One of the side effects of using the detection scheme in Fig. 1 is that there is a significant signal at the central frequency for the spectrum, $f_{\mathrm{IF}}$, resulting from the downconversion necessary for signal recording with a moderate bandwidth $(3.5 \mathrm{GHz})$ oscilloscope. This unwanted signal is fortunately a constant amplitude signal of exactly known frequency so that it is efficiently removed in the time domain by fitting and subtracting a suitable sine-wave. More general time domain filtering features, as pioneered in the Kiel laboratory [22], have also been built into the program, so that selected constant amplitude or decaying spurious signals can be removed from the frequency domain.

Another difficulty in using the scheme employing many microwave pulses per one gas pulse lies in establishing the suitable delay time between the opening of the supersonic expansion nozzle and commencement of microwave pulsing. The sample response to successive exciting microwave pulses may also vary due to backing pressure and mechanical adjustment of the expansion nozzle so that its knowledge is useful for optimising the experiment. With a double downconversion scheme employed in the early version of our cavity FTMW spectrometer the performance of the nozzle could be assessed by direct visual inspection of the signal on the oscilloscope (see Fig. 2 of Ref. [3]). With chirped pulse spectra this is only possible for spectra containing very strong lines, since the FID signal at the early stages of averaging normally has the appearance of a relatively uniform high-frequency noise band. The solution, built into the FFTS program, has been to carry out a separate FFT transformation for every pulse in the train of microwave pulses applied to a single gas pulse. A selected segment (snippet) of each such transform is then combined into a spectrum containing all successive snippets. An example of such a snippet spectrum is reproduced in Fig. 4 and shows that 
the spectrum in Fig. 3 is centered on the maximum of the signal available from the gas expansion, which takes place at the 10th exciting microwave pulse, approximately $580 \mu \mathrm{s}$ from the beginning of the measurement.

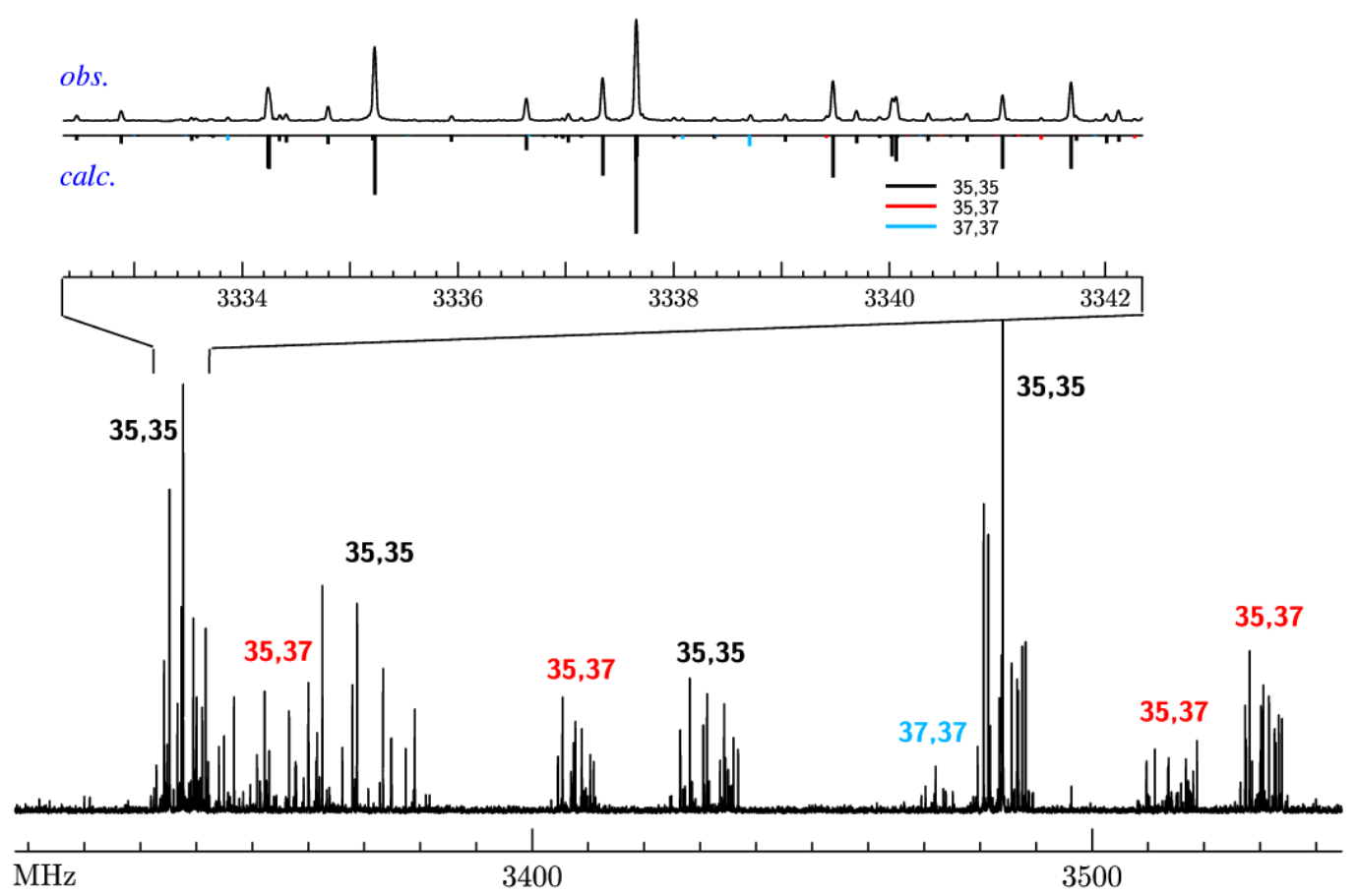

Fig. 3. Illustration of the performance of the chirped pulse spectrometer on a $300 \mathrm{MHz}$ segment of the spectrum of 2,2-dichloropropane. The chlorine isotopic composition is marked above each hyperfine multiplet, which corresponds to a single rotational transition.

The FFTS program contains many different FFT transformation and diagnostic options, as well as features for integrating chirped pulse measurements with the AABS package for Assignment and Analysis of Broadband Spectra [23, 24]. The importance of a suitable FFT transformation mode has been demonstrated in [11] where it was shown that the optimum approach for very high signal to noise $(\mathrm{S} / \mathrm{N})$ chirped pulse spectra with lines differing by many orders of magnitude in intensity is to use the Kaiser-Bessel windowing function. Furthermore, display of the amplitude of the transformed signal ensures correct relative intensities for the measured lines. The performance of this type of transformation for weak lines in a somewhat lower $\mathrm{S} / \mathrm{N}$ signal is shown in the top part of Fig. 5, where the Kaiser-Bessel windowing is at a cost of significant line broadening, to over $100 \mathrm{kHz}$. An alternative for such lines is to use a standard Bartlett windowing function for the FFT and power display of the spectrum. In this case the linewidths drop to less than $30 \mathrm{kHz}$ and there is much better separation from the noise floor (lower part of Fig. 5), but this is obtained at the cost of foregoing accurate relative intensities. Tradeoffs like these are possible with the FFTS program, which is freely available from the PROSPE website $[25,26]$ and has already been tested on chirped pulse spectra from several laboratories.

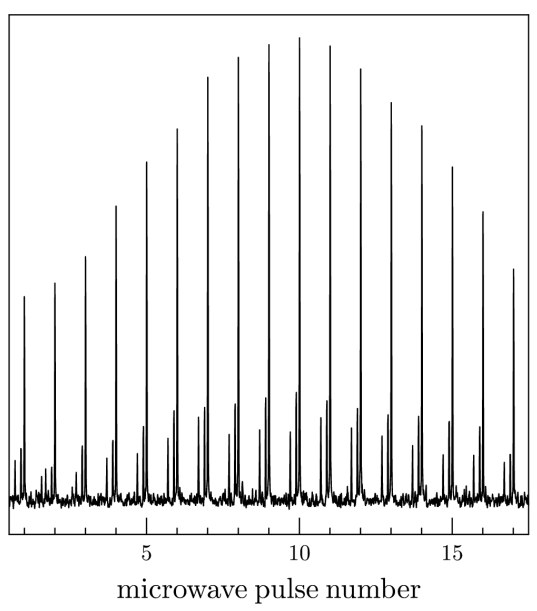

Fig. 4. Snippet spectrum derived from the FID of the spectrum in Fig. 3 and consisting of back-to-back frequency domain segments derived for successive exciting microwave pulses probing a single gas pulse. The snippets are in this case of $1 \mathrm{MHz}$ width, are centred on $3484.08 \mathrm{MHz}$, and correspond to 17 successive probing microwave pulses applied over $1 \mathrm{~ms}$, commencing $2700 \mu$ s from the beginning of the pulse opening the expansion valves. 


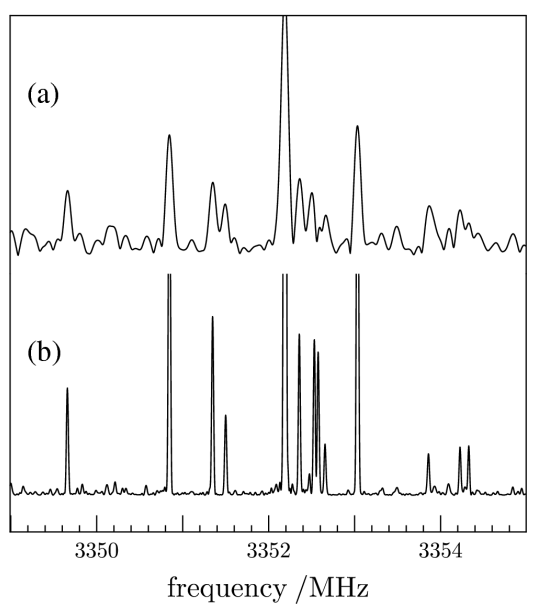

Fig. 5. Illustration of the effect of the windowing function and display mode dependence of the FFT derived spectra: (a) using Kaiser-Bessel windowing function and amplitude display and (b) using the Bartlett windowing function and power display.

\section{Rotational spectroscopy}

\subsection{Identification of a new molecular species}

In one of the spectra of DCP, shown in Fig. 6, we noted the presence of a characteristic triplet of lines just outside the frequency region covered by the employed chirp pulse. The fact that those lines were visible at the much decreased excitation power available from the harmonics of the nominal chirp suggested that they were quite intense. This was confirmed by a spectrum covering the region of the unexpected lines, reproduced in Fig. 7.

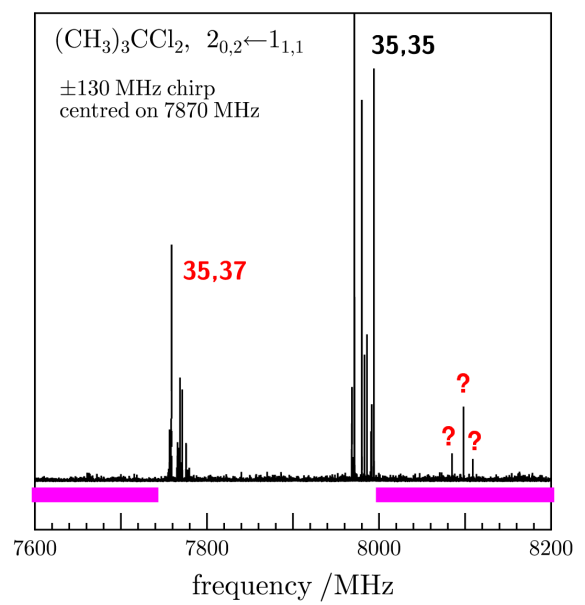

Fig. 6. The chirped pulse spectrum recorded in order to measure the $2_{0,2} \leftarrow 1_{1,1}$ rotational transition of DCP. The thick horizontal lines indicate regions outside the nominal chirped pulse coverage, where transitions can also be observed, but at much reduced excitation power. The presence of an unexpected triplet of transitions near $8100 \mathrm{GHz}$ suggests their considerable intensity.

This was quite a surprising development since the new lines were considerably stronger than those of DCP. Observation of triplets suggested that those belonged to a $J=1 \leftarrow 0$ rotational transition of a molecule with a single quadrupolar atom. The presence of two such triplets, their relative intensity and width, pointed to chlorine as the quadrupolar atoms. Exploration of the frequency region at twice that measured in Fig. 7 revealed another multiplet with a hyperfine splitting pattern of a $J=2 \leftarrow 1$ transition, and it did not take long to identify the full set of ${ }^{a} R$-branch $J=2 \leftarrow 1$ transitions. The four transitions showed that the carrier molecule is a prolate type rotor and predictions showed that only one more transition, $3_{1,2} \leftarrow 3_{1,3}$, was to be expected near $10 \mathrm{GHz}$. This transition was observed, confirming the spectroscopic, though not yet a chemical, identification of the new species. There was now more than sufficient information for determination of values of the three rotational constants, and of the inertia defect, $\Delta_{i}=I_{c}-I_{a}-I_{b}=-3.0 \mu \AA^{2}$. This value is characteristic of a near planar molecule with a single methyl group positioned in a way such that two of the hydrogens are out of the molecular plane.

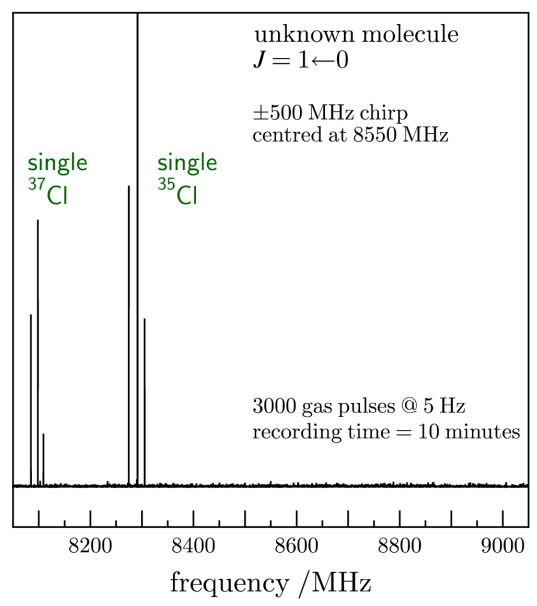

Fig. 7. Chirped pulse spectrum covering the new transitions. The hyperfine splitting structure is characteristic of a $J=1 \leftarrow 0$ rotational transition of a molecule containing a single chlorine nucleus.

Consideration of several close chemical relatives of DCP revealed that the only one fulfilling the constraints imposed by the unknown lines in the chirped pulse spectrum is 2-chloropropene (2CP), and the two molecules are compared in Fig. 8.

\subsection{Spectroscopic constants for 2-chloropropene}

Once the unknown molecule was tentatively identified as $2 \mathrm{CP}$ we looked for literature data and found that there were two previous studies of $2 \mathrm{CP}$ by rotational spectroscopy $[27,28]$. The literature spectroscopic constants are compared in the first two columns of Table I with those of our unknown molecule as derived from fitting only the five hyperfine multiplets identified by chirped pulse spectroscopy. The comparison unambiguously confirms the assignment of the new species as $2 \mathrm{CP}$, even though there are some numerical discrepancies in the values of constants due to fitting small data sets. 

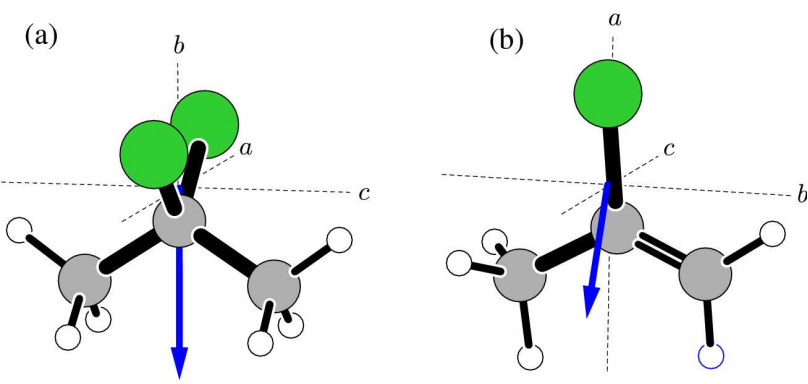

Fig. 8. The orientations in the inertial axes and the relative magnitudes and directions of the electric dipole moments for the two molecules measured in this work: (a) 2,2-dichloropropane, (b) 2-chloropropene.

TABLE I

Comparison of spectroscopic constants between the initially unknown molecule identified in the chirped pulse spectrum and 2-chloropropene.

\begin{tabular}{l|c|c|c|c}
\hline \hline & unknown & \multicolumn{3}{|c}{ 2-chloropropene } \\
\cline { 2 - 5 } & chirped pulse & Ref. [28 $^{a}$ & global fit $^{b}$ & calc. $^{c}$ \\
\hline$A[\mathrm{MHz}]$ & $9272.118(36)^{d}$ & $9271.853(54)$ & $9271.8627(15)$ & \\
$B[\mathrm{MHz}]$ & $4981.8219(30)$ & $4983.841(31)$ & $4983.82695(26)$ & \\
$C[\mathrm{MHz}]$ & $3304.3884(30)$ & $3304.391(31)$ & $3304.38202(21)$ & \\
$\Delta_{J}[\mathrm{kHz}]$ & $1.99(25)$ & $-0.9(41)$ & $1.49826(11)$ & 1.466 \\
$\Delta_{J K}[\mathrm{kHz}]$ & {$[0]$.} & $2.3(20)$ & $3.38533(78)$ & 3.305 \\
$\Delta_{K}[\mathrm{kHz}]$ & {$[0]$.} & $6.8(55)$ & $3.228(10)$ & 3.146 \\
$\delta_{J}[\mathrm{kHz}]$ & $0.65(10)$ & $0.47(11)$ & $0.534603(43)$ & 0.514 \\
$\delta_{K}[\mathrm{kHz}]$ & {$[0]$.} & $5.3(15)$ & $4.1949(14)$ & 4.094 \\
$\Phi_{K}[\mathrm{~Hz}]$ & & & $-0.326(13)$ & \\
& & & & \\
$\chi_{a a}[\mathrm{MHz}]$ & $-68.1320(67)$ & $-68.134(14)$ & $-68.1308(59)$ & \\
$\chi_{b b}[\mathrm{MHz}]$ & $37.1696(83)$ & $37.163(17)$ & $37.1638(67)$ & \\
$\chi_{c c}[\mathrm{MHz}]$ & $30.9624(83)$ & $30.971(17)$ & $30.9670(67)$ & \\
$\Delta_{i}\left[\mu \mathrm{uA} \AA^{2}\right]$ & $-3.0081(3)$ & $-2.969(2)$ & $-2.96848(2)$ & \\
$\sigma_{\text {fit }}[\mathrm{kHz}]$ & 4.25 & 46.9 & 29.2 & \\
$N_{\text {lines }}$ & 24 & 27 & 202 & \\
$\sigma_{\text {not }}$ & & & &
\end{tabular}

${ }^{a}$ Rotational and centrifugal distortion constants are from a refit of hyperfine-free transitions from Ref. [28] with Watson's $A$-reduced Hamiltonian [29].

${ }^{b}$ Weighted fit of chirped pulse and MMW measurements made in this work, combined with data from Ref. [28], resulting in unitless deviation of fit $\sigma_{\mathrm{rms}}=0.86$.

${ }^{c}$ Evaluated from an unscaled MP2 $6311 \mathrm{G}++(\mathrm{d}, \mathrm{p})$ harmonic force field, obtained with FIREFLY [30].

${ }^{d}$ The quantities in round parentheses are standard errors in units of the last quoted digit of the value of the constant.

At that stage we combined the chirped pulse data with those from Ref. [28] in order to check for possible transitions of $2 \mathrm{CP}$ in the legacy $\mathrm{mm}$ wave spectrum of DCP used for the results reported in Ref. [21]. We were readily able to identify 36 different ${ }^{a} R$ - branch transitions with values of $K_{a}$ from 0 to 6 . Those were at relatively erratic relative intensities in relation to the DCP lines, presumably being a function of how much of the more volatile $2 \mathrm{CP}$ was already distilled off the sample. We then used a fresh sample of DCP and measured 53 transitions of $2 \mathrm{CP}$ at frequencies between 272 and $311 \mathrm{GHz}$, and for significantly higher values of $K_{a}$, up to 26 . The higher $K_{a}$ transitions were split into internal rotation doublets and in such cases we used the mean frequency of the doublet.
The resulting global fit of all measurements is presented in the penultimate column of Table I. Complete data files are available in the data section of the PROSPE website. The final column of Table I lists $a b$ initio calculated quartic centrifugal distortion constants for $2 \mathrm{CP}$, which are in excellent agreement with those determined presently. We note that the highest frequency rotational transition measured for 2CP near $18260 \mathrm{MHz}$, as shown in Fig. 9, confirms the usefulness of the new chirped pulse spectrometer also near the upper frequency limit of the design. The global fit of transitions for $2 \mathrm{CP}$ reported in Table I, gives insight into the frequency measurement precision of the new spectrometer. This turns out to be less than $5 \mathrm{kHz}$, which is similar to the values obtained recently for two different supersonic expansion, chirped pulse spectrometers $[31,32]$.

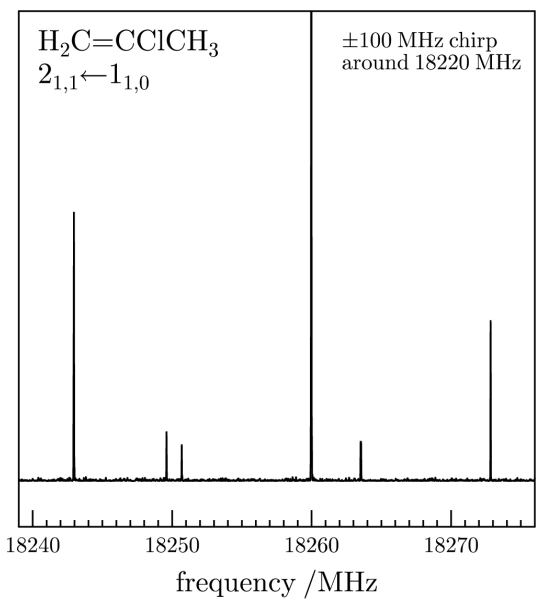

Fig. 9. A segment of the chirped pulse spectrum covering the $2_{1,1} \leftarrow 1_{1,0}$ rotational transition of 2 chloropropene illustrating the performance of the new spectrometer at the top of its design frequency coverage.

\section{Conclusions}

The present work reports the design and operation of a new chirped pulse spectrometer for measuring supersonic expansion rotational spectra at $2-18 \mathrm{GHz}$. Various instrumental challenges have been overcome and the spectrometer is accompanied by a new computer program for FFT analysis that is being made available to the spectroscopic community. The commissioning phase of the spectrometer allowed an accidental discovery of a significant impurity in an intensively studied sample of the DCP molecule that was overlooked by other methods. Although we used a commercial sample of $98 \%$ nominal purity there was considerable enrichment in $2 \mathrm{CP}$ when evaporated from a liquid room temperature sample, and even more from a sample previously cooled with liquid nitrogen and allowed to slowly warm up. In the latter conditions we estimate from the $\mathrm{mm}$ wave spectrum that $2 \mathrm{CP}: \mathrm{DCP}$ ratio of 1:2 could be achieved, which is not surprising since the boiling point of $2 \mathrm{CP}$ is $23^{\circ} \mathrm{C}$, while that of DCP is $70^{\circ} \mathrm{C}$. $2 \mathrm{CP}$ appears to be a chemical 
precursor to DCP since the two are related by addition of $\mathrm{HCl}$ across the $2 \mathrm{CP}$ double bond. As a result of this accidental discovery we are presently able to report the most precise spectroscopic constants for $2 \mathrm{CP}$ that are of sufficient quality for possible environmental monitoring of this molecule.

\section{Acknowledgments}

Dr Nick Walker from Newcastle University (UK) is thanked for help with attenuating the spectrometer chamber resonances, and Prof. Brooks Pate of Virginia University (US) is thanked for sharing his ideas on chirped pulse spectrometer design. The authors acknowledge financial support from a grant from the Polish National Science Centre, decision number DEC/2011/02/A/ST2/00298.

\section{References}

[1] T.J. Balle, W.H. Flygare, Rev. Sci. Instrum. 52, 33 (1981).

[2] W. Caminati, J.-U. Grabow, in: Frontiers of Molecular Spectroscopy, Ed. J. Laane, Elsevier, 2009, p. 455.

[3] Z. Kisiel, J. Kosarzewski, L. Pszczółkowski, Acta. Phys. Pol. A 92, 507 (1997).

[4] Z. Kisiel, E. Białkowska-Jaworska, L. Pszczółkowski, A. Milet, C. Struniewicz, R. Moszynski, J. Sadlej, J. Chem. Phys. 112, 5767 (2000).

[5] Z. Kisiel, B.A. Pietrewicz, O. Desyatnyk, L. Pszczółkowski, I. Struniewicz, J. Sadlej, J. Chem. Phys. 119, 5907 (2003).

[6] Z. Kisiel, L. Pszczółkowski, J.C. López, J.L. Alonso, A. Maris, W. Caminati, J. Mol. Spectrosc. 195, 332 (1999).

[7] O. Desyatnyk, L. Pszczółkowski, S. Thorwirth, T.M. Krygowski, Z. Kisiel, Phys. Chem. Chem. Phys. 7, 1708 (2005).

[8] Z. Kisiel, O. Desyatnyk, E. Białkowska-Jaworska, L. Pszczółkowski, Phys. Chem. Chem. Phys. 5, 820 (2003).

[9] G.G. Brown, B.C. Dian, K.O. Douglass, S.M. Geyer, S.T. Shipman, B.H. Pate, Rev. Sci. Instrum. 79, 053103 (2008).

[10] J.L. Neill, S.T. Shipman, L. Alvarez-Valtierra, A. Lesarri, Z. Kisiel, J. Mol. Spectrosc. 269, 21 (2011).

[11] C. Pérez, S. Lobsiger, N.A. Seifert, D.P. Zaleski, B. Temelso, G.C. Shields, Z. Kisiel, B.H. Pate, Chem. Phys. Lett. 571, 1 (2013).

[12] C. Pérez, M.T. Muckle, D.P. Zaleski, N.A. Seifert, B. Temelso, G.C. Shields, Z. Kisiel, B.H. Pate, Science 336, 897 (2012).

[13] C. Pérez, D.P. Zaleski, N.A. Seifert, B. Temelso, G.C. Shields, Z. Kisiel, B.H. Pate, $A n$ gew. Chem. Int. Ed. 53, 14368 (2014).
[14] J.O. Richardson, C. Pérez, S. Lobsiger, A.A. Reid, B. Temelso, G.C. Shields, Z. Kisiel, D.J. Wales, B.H. Pate, S.C. Althorpe, Science 351, 1310 (2016).

[15] M.D. Marshall, H.O. Leung, B.Q. Scheertz, J.E. Thaler, J.S. Muenter, J. Mol. Spectrosc. 266, 37 (2011).

[16] L. Evangelisti, G. Sedo, J. van Wijngaarden, J. Phys. Chem. A 115, 685 (2011).

[17] G.S. Grubbs II, C.T. Dewberry, K.C. Etchison, K.E. Kerr, S.A. Cooke, Rev. Sci. Instrum. $\mathbf{7 8}$, 096106 (2007).

[18] G.S. Grubbs II, R.A. Powoski, D. Jojola, S.A. Cooke, J. Phys. Chem. A 114, 8009 (2010).

[19] D.A. Obenchain, A.A. Elliott, A.L. Steber, R.A. Peebles, S.A. Peebles, C.J. Wurrey, G.A. Guirgis, J. Mol. Spectrosc. 261, 35 (2010).

[20] Z. Kisiel, J. Kosarzewski, B.A. Pietrewicz, L. Pszczółkowski, Chem. Phys. Lett. 325, 523 (2000).

[21] E. Białkowska-Jaworska, L. Pszczółkowski, Z. Kisiel, J. Mol. Spectrosc. 308-309, 20 (2015).

[22] J. Haeckel, H. Mäder, Z. Nat.forsch. A 43, 203 (1988).

[23] Z. Kisiel, L. Pszczółkowski, I.R. Medvedev, M. Winnewisser, F.C. De Lucia, E. Herbst, J. Mol. Spectrosc. 233231 (2005).

[24] Z. Kisiel, L. Pszczółkowski, B.J. Drouin, C.S. Brauer, S. Yu, J.C. Pearson, I.R. Medvedev, S. Fortman, C. Neese, J. Mol. Spectrosc. 280134 (2012).

[25] Z. Kisiel, in: Spectroscopy from Space, Eds. J. Demaison, K. Sarka, E.A. Cohen, Kluwer Academic, Dordrecht 2001, p. 91.

[26] Z. Kisiel, PROSPE - Programs for ROtational SPEctroscopy, Institute of Physics, Polish Academy of Sciences, Warsaw.

[27] M.L. Unland, V. Weiss, W.H. Flygare, J. Chem. Phys. 422138 (1965).

[28] F. Fliege, H. Dreizler, Z. Nat.forsch. A 38, 1231 (1983).

[29] J.K.G. Watson, in: Vibrational Spectra and Structure, Ed. J.R. Durig, Vol. 6, Elsevier, New York 1977, p. 1.

[30] A.A. Granovsky, Firefly version 8 computing package, based on the Gamess-US package, M.W.Schmidt, K.K. Baldridge, J.A. Boatz, S.T. Elbert, M.S. Gordon, J.H. Jensen, S. Koseki, N. Matsunaga, K.A. Nguyen, S. Su, T.L. Windus, M. Dupuis, J.A. Montgomery, J. Comput. Chem. 14, 1347 (1993).

[31] C. Pérez, A.L. Steber, J.C. López, Z. Kisiel, M. Schnell, J. Phys. Chem. Lett. 7, 154 (2016).

[32] I. Uriarte, Z. Kisiel, E. Białkowska-Jaworska, L. Pszczółkowski, P. Ecija, F.J. Basterretxea, E.J. Cocinero, J. Mol. Spectrosc., (2017). 